\title{
The relationship between lower urinary tract dysfunctions and urinary leakage from ureterocystoneoanastomosis in male patients after kidney transplantation
}

\author{
Zachoval $\mathrm{R}^{1}$, Krhut $\mathrm{J}^{2}$, Slatinska $\mathrm{J}^{3}$, Viklicky $\mathrm{O}^{3}$, Janousek $\mathrm{L}^{4}$ \\ Department of Urology, 3rd Faculty of Medicine of Charles Univeristy and Thomayer Hospital, \\ Prague, Czech Republic. roman.zachoval@ftn.cz
}

\begin{abstract}
OBJECTIVES: The aim of this study was to investigate the association of lower urinary tract dysfunctions with urinary leakage from ureterocystoneoanastomosis (UCNA) after kidney transplantation.

BACKGROUND: The UCNA leakage after kidney transplantation can be associated with various conditions while severe lower urinary tract dysfunctions could be one of them.

METHODS: The analysis included all men who underwent kidney transplantation between January 2009 and December 2014. The parameters of storage and voiding functions were evaluated. All patients were monitored during their post-transplantation period for the incidence of urinary leakage from UCNA. Urodynamic parameters were compared between men with and without a documented leakage.

RESULTS: The study cohort included 127 male patients, while UCNA leakage was observed in 11 (8.7 \%) patients. Significant differences between both groups of patients were found for storage parameters (patients with leakage had smaller volume at first and a normal desire to void, smaller maximal cystometric capacity, and lower detrusor compliance) and voiding parameters (patients with leakage had a lower maximal flow rate, higher detrusor pressure at maximal flow rate and higher bladder outlet obstruction index).

CONCLUSION: This study shows an association between lower urinary tract dysfunction and UCNA leakage in men without previous urological history (Tab. 2, Fig. 2, Ref. 24). Text in PDF www.elis.sk

KEY WORDS: urinary leakage, ureterocystoneoanastomosis, lower urinary tract dysfunctions, kidney transplantation.
\end{abstract}

\section{Introduction}

The number of kidney transplants steadily increases in countries worldwide, including the Czech Republic (1). This is due to several factors, mainly owing to the improvements in care during pre-transplant, peri-operative and post-transplant periods, which made it possible to carry out kidney transplantations in patients also in cases where the conditions of donor or recipient would have precluded such a procedure in the past. New methods have brought about an improvement in transplantation outcomes, albeit posttransplant complications still occur. They lead to graft rejection, graft function impairment, or put the patient's life at risk $(2,3)$.

${ }^{1}$ Department of Urology, 3rd Faculty of Medicine of Charles Univeristy and Thomayer Hospital, Prague, Czech Republic, ${ }^{2}$ Department of Urology, University Hospital in Ostrava and Faculty of Medicine of University of Ostrava, Ostrava, Czech Republic, ${ }^{3}$ Department of Nephrology, Institute of Clinical and Experimental Medicine, Prague, Czech Republic, and ${ }^{4}$ Department of Transplantation Surgery, Institute of Clinical and Experimental Medicine, Prague, Czech Republic

Address for correspondence: R. Zachoval, Prof, MD, PhD, Department of Urology, Thomayer Hospital, Videnska 800, CZ-140 59 Prague 4, Czech Republic.

Phone: +420.261083196 , Fax: +420.261083688
Urinary leakage from the ureterocystoneoanastomosis (UCNA) is a complication of kidney transplantation that occurs in 1.2-6.2\% of patients (4). Several potential causes have been identified, in particular inappropriate anastomotic technique and distal ureteric ischaemia. Other factors might include lower urinary tract (LUT) dysfunctions such as detrusor overactivity or bladder outlet obstruction. Both may cause higher intravesical pressures leading to mechanical distress of UCNA, bladder wall structural changes (fibrosis, decrease in vascularisation) and subsequently impaired UCNA healing (5).

The primary objective of our study was to investigate different LUT dysfunctions in relation to the occurrence of UCNA leakage in male patients after kidney transplantation. The secondary objective was to determine which specific parameters are associated with the risk of urinary leakage from UCNA and their respective cut-off values.

\section{Materials and methods}

The analysis included all men who underwent kidney transplantation between January 2009 and December 2014 at our centre and who exhibited normal findings (except for reduced urine production) on pre-transplant urological assessment. 
The following parameters were recorded for all subjects:

LUT function evaluated by urodynamic examination according to the Good Urodynamic Practice recommendations (6):

a. Storage function parameters as determined by filling cystometry

i. first desire to void (FDV) $(\mathrm{mL})$

ii. normal desire to void (NDV) $(\mathrm{mL})$

iii. maximal cystometric capacity $(\mathrm{Cmax})$ (normalvalue $>350 \mathrm{~mL}$ )

iv. detrusor compliance (normal value $>30$ )

v. maximal detrusor pressure (Pdet.max) $\left(\mathrm{cm} \mathrm{H}_{2} \mathrm{O}\right)$

vi. presence of uninhibited detrusor contractions

b. Voiding parameters as documented by voiding cystometry

i. maximal flow rate (Qmax.p) $(\mathrm{mL} / \mathrm{s})$

ii. post-void residual measured by ultrasound $(\mathrm{mL})$

iii. detrusor pressure at maximal flow rate (PdetQmax) $(\mathrm{cm}$ $\mathrm{H}_{2} \mathrm{O}$ )

iv. bladder outlet obstruction index $(\mathrm{BOOI}=\mathrm{PdetQmax}-2 \times$ Qmax) (normal value $<40$ )

v. bladder contractility index $(\mathrm{BCI}=\mathrm{PdetQmax}+5 \times \mathrm{Qmax})$ (normal value $>100$ )

The following patients were excluded from the study:

- those with the history of LUT surgery (e.g., transurethral resection of the prostate)

- those with urine output smaller than $500 \mathrm{~mL} / 24$ hours

- those with moderate or serious voiding difficulties as evidenced by International Prostate Symptom Score questionnaire (i.e., IPSS $>7$ on a scale of $0-35$ )

- those with urological diseases that might have effects on LUT (e.g., bladder cancer, urinary bladder stones, etc.)

- those with a maximal flow rate (Qmax) slower than $15 \mathrm{~mL} / \mathrm{s}$ on uroflowmetry (assessed only in patients with daily urine production $>500 \mathrm{~mL}$ ); minimum voided volume was set at $150 \mathrm{~mL}$

- those on medical treatment for LUT symptoms

- those who suffered from neurological diseases or other diseases that might have an impact on LUT functions (e.g., diabetes mellitus, cerebral stroke, spinal lesion, etc.)

All patients were monitored during the post-transplantation period for the incidence of urinary leakage from UCNA. Urodynamic parameters were compared between those with and without UCNA leakage.

\section{Statistical analysis}

Statistical analysis was performed using SW SAS (SAS Institute Inc., Cary, NC, USA) and SW STATISTICA (StatSoft, Inc., Tulsa, OK, USA).

Descriptive statistics such as mean, standard deviation, variance, median, interquartile range (IQR), minimum and maximum were compared between the two groups. The Wilcoxon two-sample test was used to compare differences in proportions. Spearman's correlation coefficients were used to test correlations between variables. $\chi 2$ test and Fisher's exact test (for sparse data) were used to test differences in proportions. Specificity, sensitivity, ROC curves and odds-ratios with $95 \%$ confidence intervals were computed for relevant variables.

All tests were carried out with an $\alpha$-level of 0.05 .

\section{Results}

The study population included 127 male patients who had been given complete information on the analysed parameters. The median age was 59 years.

The cause of chronic kidney disease (CKD) was glomerulonephritis, hypertension nephropathy, diabetic nephropathy, tubulointerstital nephritis, IgA nephropathy, renal polycystosis, focal segmental glomerulosclerosis, and other or unclear reasons in 21 , $20,19,19,12,12,4$ and 20 patients, respectively. UCNA leakage occurred in $11(8.7 \%)$ patients.

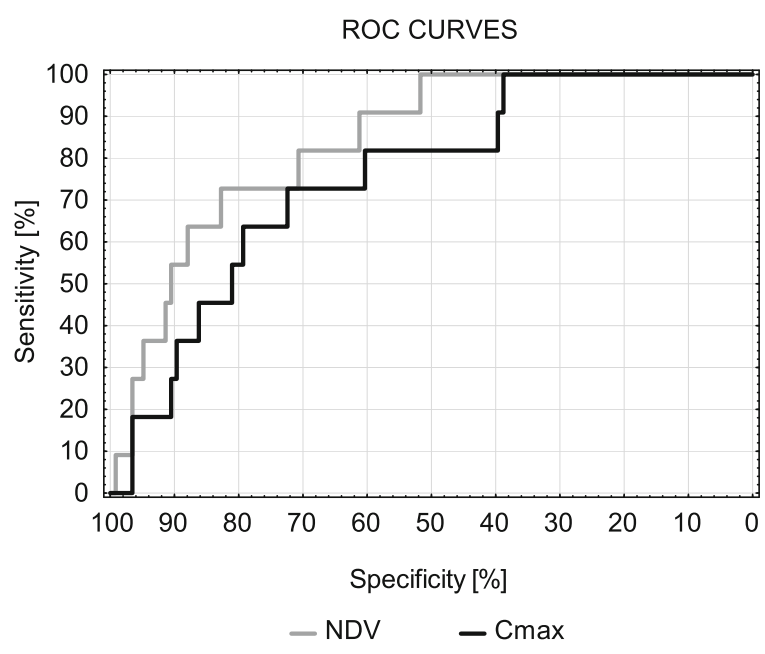

Fig. 1. ROC curves for NDV and Cmax.

ROC CURVES

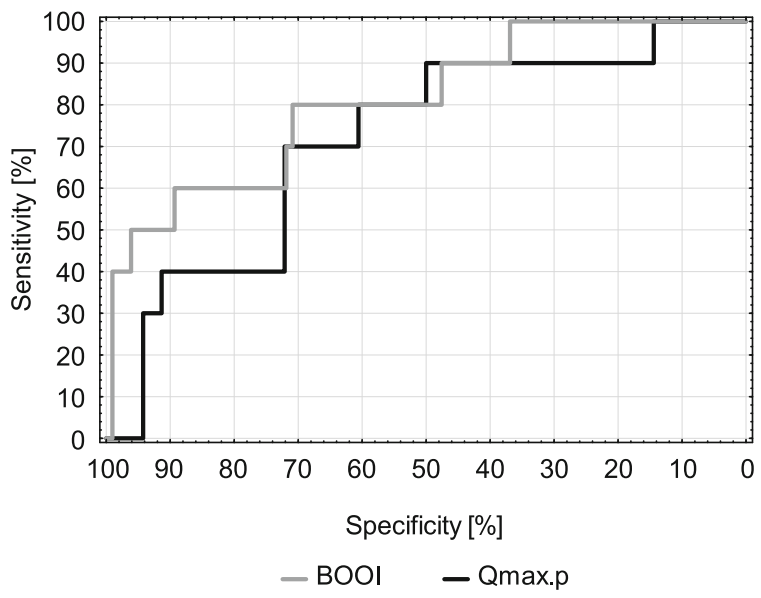

Fig. 2. ROC curves for Qmax.p and BOOI. 


\section{$336-340$}

Storage and voiding function parameters of the study population determined by urodynamic examination are summarized in Table 1.

The comparisons of urodynamic parameters in patients with and without urinary leakage are shown in Table 2. Significant differences between men with and without UCNA leakage were demonstrated in storage function parameters as follows: FDV, NDV, Cmax and compliance; and in voiding function parameters as follows: Qmax.p, PdetQmax and BOOI. The ROC curves for the most frequently used filling and voiding function parameters are demonstrated in Figures 1 and 2. Uninhibited detrusor contractions were present in 51 patients $(40 \%)$.

Tab. 1. Results of urodynamic examinations in all patients and patients with and without urinary leak.

\begin{tabular}{|c|c|c|c|c|c|}
\hline \multicolumn{6}{|l|}{ All patients } \\
\hline Parameters & Minimum & Lower Quartile & Median & Upper Quartile & Maximum \\
\hline FDV & 5.00 & 56.00 & 89.00 & 137.00 & 391.00 \\
\hline NDV & 26.00 & 98.00 & 138.00 & 213.00 & 691.00 \\
\hline Cmax & 22.00 & 144.00 & 211.00 & 341.00 & 914.00 \\
\hline compliance & 0.20 & 4.45 & 14.10 & 32.45 & 320.00 \\
\hline Pdet.max & 2.00 & 17.00 & 35.00 & 65.00 & 221.00 \\
\hline Qmax & 1.00 & 4.00 & 6.00 & 11.00 & 26.00 \\
\hline PVR & 0.00 & 0.00 & 0.00 & 97.00 & 924.00 \\
\hline PdetQmax & 12.00 & 35.00 & 52.00 & 72.00 & 163.00 \\
\hline BOOI & 20.00 & 15.00 & 37.00 & 60.00 & 155.00 \\
\hline BCI & 36.00 & 76.00 & 92.00 & 113.00 & 183.00 \\
\hline \multicolumn{6}{|c|}{ Patients with leak } \\
\hline Parameters & Minimum & Lower Quartile & Median & Upper Quartile & Maximum \\
\hline FDV & 8.00 & 33.00 & 60.00 & 79.00 & 135.00 \\
\hline NDV & 36.00 & 51.00 & 79.00 & 110.00 & 143.00 \\
\hline Cmax & 64.00 & 101.00 & 135.00 & 190.00 & 280.00 \\
\hline compliance & 0.60 & 2.20 & 3.40 & 19.30 & 188.00 \\
\hline Pdet.max & 11.00 & 40.00 & 57.50 & 92.00 & 221.00 \\
\hline Qmax & 1.00 & 2.00 & 4.00 & 5.00 & 14.00 \\
\hline PVR & 0.00 & 0.00 & 0.00 & 40.00 & 360.00 \\
\hline PdetQmax & 39.00 & 56.00 & 93.00 & 134.00 & 145.00 \\
\hline BOOI & 24.00 & 50.00 & 85.00 & 128.00 & 143.00 \\
\hline BCI & 59.00 & 85.00 & 124.00 & 149.00 & 163.00 \\
\hline
\end{tabular}

\begin{tabular}{lccccc}
\hline Patients without leak & \multicolumn{5}{c}{} \\
\hline Parameters & Minimum & Lower Quartile & Median & Upper Quartile & Maximum \\
\hline FDV & 5.00 & 57.00 & 93.00 & 147.00 & 391.00 \\
NDV & 26.00 & 107.00 & 147.00 & 227.00 & 691.00 \\
Cmax & 22.00 & 150.50 & 227.50 & 356.00 & 914.00 \\
compliance & 0.20 & 5.00 & 14.50 & 36.70 & 320.00 \\
Pdet.max & 2.00 & 16.00 & 29.00 & 61.00 & 140.00 \\
Qmax & 1.00 & 4.00 & 6.50 & 11.00 & 26.00 \\
PVR & 0.00 & 0.00 & 0.00 & 98.00 & 924.00 \\
PdetQmax & 12.00 & 34.00 & 48.00 & 66.00 & 163.00 \\
BOOI & 20.00 & 14.00 & 33.00 & 58.00 & 155.00 \\
BCI & 36.00 & 76.00 & 91.00 & 110.00 & 183.00 \\
\hline
\end{tabular}

\section{Discussion}

Our results show for the first time a relationship between LUT dysfunctions and UCNA leakage after kidney transplantation. The incidence of UCNA leakage is significantly higher in patients with a hypersensitive, overactive detrusor (who have decreased bladder volumes at FDV, NDV, decreased Cmax and detrusor compliance) as well as in patients with impaired voiding function (decreased Qmax and PdetQmax, increased BOOI).

Patients with CKD have got a high incidence of LUT symptoms for a variety of reasons. Not all of the etiologic factors have been elucidated so far; the following ones are considered to be the most important: reduced urinary output, difference in urine composition, chronic inflammation and LUT ischaemia, lack of protective compounds in urine, changes in neuroreceptors and reflex functioning of the LUT due to uraemic neuropathy, and duration of CKD (7-9). For reasons listed above and/or due to other LUT abnormalities (such as benign prostate hyperplasia (BPH), overactive bladder, aging-related degenerative changes of the bladder or bladder outlet), LUT of CKD patients undergoes morphological changes, e.g., fibrosis, detrusor muscle remodelling or perineural fibrosis (5).

CKD seems to be associated with both storage and voiding LUT dysfunction. Zer-

Tab. 2. Comparison of urodynamic parameters in patients with and without urinary leak.

\begin{tabular}{|c|c|c|c|c|c|c|c|}
\hline Parameter & $\mathrm{p}$ & Cut-off value & Sensitivity (\%) & Specificity (\%) & Odds-ratio & AUC & $95 \%$ confidence interval \\
\hline FDV & 0.0144 & $75 \mathrm{~mL}$ & 72.7 & 69.3 & 4.5 & 0.74 & $0.59-0.88$ \\
\hline NDV & 0.0003 & $110 \mathrm{~mL}$ & 81.8 & 70.7 & 8.4 & 0.83 & $0.72-0.94$ \\
\hline Cmax & 0.006 & $153 \mathrm{~mL}$ & 72.7 & 72.4 & 4.5 & 0.79 & $0.67-0.91$ \\
\hline compliance & 0.0402 & 5 & 70.0 & 73.7 & 6.0 & 0.78 & $0.63-0.94$ \\
\hline Pdet.max & 0.0504 & - & - & - & - & & \\
\hline Qmax.p & 0.0059 & $5 \mathrm{~mL} / \mathrm{s}$ & 80.0 & 60.6 & 6.1 & 0.78 & $0.63-0.94$ \\
\hline PVR & 0.465 & - & - & - & - & & \\
\hline BOOI & 0.0018 & $50^{2}$ & 80.0 & 70.0 & 6.5 & 0.80 & $0.65-0.95$ \\
\hline BCI & 0.064 & - & - & - & - & & \\
\hline
\end{tabular}


mann et al reported that $38 \%$ of patients with CKD were at risk of low bladder compliance, and $48 \%$ were at risk of detrusor hyperactivity (10). Tsunoyama et al found that patients with end-stage kidney disease (ESKD) and reduced urine output suffered from reduced FS, NS and SS as compared to the normal population, and $27 \%$ had detrusor hyperactivity (10). Chen et al reported LUT obstruction in $51 \%$ of patients with CKD (8).

Previous studies showed that morphological and functional changes in LUT depend on the duration of CKD (5). According to Martin et al, detrusor capacity decreases to $300 \mathrm{~mL}$ after 5 years and to $150 \mathrm{~mL}$ after 15 years in patients with ESKD (9). Dion et al reported that detrusor compliance was reduced after 1 and 10 years of dialysis in $31 \%$ and $77 \%$ of patients, respectively (12).

Increasing numbers of patients of older age groups undergo transplantations nowadays. Aging occurs at many levels and includes changes at the molecular, cellular and organ levels (such as cerebral function) and in the organism as a whole (13). LUT aging is characterized by detrusor hyperactivity, reduced detrusor contractibility and presence of subvesical obstruction. Balslev Jorgensen et al found that all uroflowmetry parameters decrease with age, even in asymptomatic individuals (14).

Studies that evaluated the occurrence of some types of LUT dysfunction and their dependence on age revealed differences between men and women. Madersbacher et al found an increase in detrusor hyperactivity that was age-dependent in men only. Among men aged 40 to 60 years, $23 \%$ exhibited detrusor hyperactivity, as did $47 \%$ of men over 80 years of age (15). Studies of voiding function have repeatedly confirmed that Qmax is reduced in men over 50 years of age $(16,17)$.

In our study population, the incidence of UCNA leakage is higher than reported elsewhere (4). This may have several causes; the presented cohort only includes males, and men have a generally higher incidence of complications after kidney transplantation. Other published studies usually evaluate urological complications irrespective of patient sex $(4,18)$. A study by Englesbe et al reported male sex to be associated with an increased incidence of complications after kidney transplantation (19), which corroborates our results. In our opinion, the main reason for the higher incidence of UCNA leakage in our population is the high proportion of patients with anuria or minimal urine output. Although the difference in urinary production was not statistically significant in men with and without UCNA leakage in our study, an association has been reported between diminished urine production, prolonged anuria, and duration of kidney replacement therapy with urodynamic deterioration (20). It implies that patients with lower residual urinary production or anuria and those on kidney replacement therapy are at higher risk of LUT deterioration and hence have a higher probability of UCNA leakage.

Future research should focus on the identification of a subgroup of patients at risk who will benefit from LUT functional assessment despite having no previous history of urological symptoms. At present, it seems reasonable to perform a quick, non-invasive functional assessment (UFM and PVR) in patients older than 50-60 years, which is when LUT dysfunctions become more prevalent, be it due to BPH or overactive bladder. A more thorough assess- ment might only be indicated in patients with long-term minimal urine production, especially in older age groups.

The relation between LUT dysfunctions and graft function remains to be determined. A unique study on correlation of urodynamic findings before kidney transplant with post-transplant renal functions and graft survival reported worse outcomes in patients with low-capacity bladders. Unfortunately, the study population was rather small (21). Another study, although not based on urodynamics, reported an association of BPH (by extension, potential LUT dysfunction) with acute urinary retention, urinary tract infection and graft loss in a population of 23,622 men with kidney transplant (22). To our knowledge, there have been no reports on the relationship between pre- and post-transplant urodynamic parameters and graft function.

In our study, we have not noticed the worsening of renal functions in patients with UCNA leakage, but the patients with graft explantation due to UCNA leakage were excluded from the follow-up, and therefore, this conclusion may be biased. UCNA leakage might have an adverse effect on graft function; this should be investigated on a larger patient population.

Our study results might have an impact on the management of a specific kidney transplant patient subgroup. Those with a significant LUT dysfunction upon preoperative examination of urodynamics and hence at risk of UCNA leakage may benefit from a modified approach as compared to those not at risk. This might entail the insertion of a JJ-stent to protect the anastomosis or a prolonged urethral catheterisation. While ureteric stenting reduces the risk of UCNA leakage, it increases the likelihood of a urinary tract infection and even a transient deterioration in renal function (23). If stenting could be limited to a selected group of patients, its benefits would outweigh the associated risks. The same applies to urethral catheterisation, which facilitates UCNA healing, but at the same time increases the UTI risk by 3-7\% per day of catheterisation (24).

\section{Conclusions}

We report on the association of LUT dysfunction with UCNA leakage in men without previous urological history. A selected subgroup of patients might benefit from pre-transplant functional LUT assessment.

\section{References}

1. Stratta RJ, Rohr MS, Sundberg AK et al. Increased Kidney Transplantation Utilizing Expanded Criteria Deceased Organ Donors with Results Comparable to Standard Criteria Donor Transplant. Ann Surg 2004; 239 (5): 688-697.

2. Zeng J, Christiansen AJ, Pooli A, Qiu F, LaGrange A. Safety and Clinical Outcomes of Robot-Assisted Radical Prostatectomy in Kidney Transplant Patients: A systematic review. J Endourol 2018; 3 (10): 935-943.

3. Breza J, Navratil P. Renal transplantation in adults. BJU Int 1999; 84 (2): 216-223.

4. Suttle T, Fumo D, Baghmanli Z, Saltzman B, Ortiz J. Comparison of Urologic Complications Between Ureteroneocystostomy and Uretero- 
$336-340$

ureterostomy in Renal Transplant: A MetaAnalysis. Exp Clin Transplant 2016; 14 (3): 276-281.

5. Tsaur I, Jones J, Melamed RJ, Blaheta RA, Gossmann J, Bentas W. Postoperative voiding dysfunction in older male renal transplant recipients. Transplant Proc 2009; 41 (5): 1615-1618.

6. Rosier PFWM, Schaefer W, Lose G et al. International Continence Society Good Urodynamic Practices and Terms 2016: Urodynamics, uroflowmetry, cystometry, and pressure-flow study. Neurourol Urodynam 2017; 36 (5): 1243-1260.

7. Zachoval R, Borovicka V, Marada T et al. The effects of diuresis, duration of dialysis and age on lower urinary tract function in urologically healthy male patients on the waiting list for kidney transplant. Urol J 2017; 15 (2): 49-54.

8. Chen JL, Lee MC, Kuo HC. Reduction of cystometric bladder capacity and bladder compliance with time in patients with end-stage renal disease. J Formos Med Assoc 2012; 111 (4): 209-213.

9. Martin X, Aboutaieb R, Soliman S, el Essawy A, Dawahra M, Lefrancois $\mathbf{N}$. The use of long-term defunctionalized bladder in renal transplantation: is it safe? Eur Urol 1999; 36 (5): 450-453.

10. Zermann DH, Janitzky A, Hohne M, Schubert J. Frequency and nocturia after successful renal transplantation: a normal situation? BJU Int 2006; 97 (3): 555-558.

11. Tsunoyama K, Ishida H, Omoto K, Shimizu T, Shirakawa H, Tanabe K. Bladder function of end-stage renal disease patients. Int J Urol 2010; 17 (9): 791-795.

12. Dion M, Cristea O, Langford S, Luke PPW, Sener A. Debilitating Lower Urinary Tract Symptoms in the Post-Renal Transplant Population Can Be Predicted Pretransplantation. Transplantation 2012; 187 (4): 589-594.

13. Breza J Jr, Zilinska Z, Bujdak P, Breza J Sr. Urological complications after kidney transplantation. Eur Urol Suppl 2009; 8: 678.
14. Balslev Jorgensen J, Jensen KME, Mogensen P. Longitudinal Observation on Normal and Abnormal Voiding in Men Over the Age of 50 Years. BJU 1993; 72 (4): 413-420.

15. Madersbacher S, Pycha A, Schatzl G, Mian C, Klingler CH, Marberger $\mathbf{M}$. The aging lower urinary tract: A comparative urodynamic study of men and women. Adult Urology 1998; 51 (2): 206-212.

16. Hald T, Horn T. The human urinary bladder in aging. Br J Urol 1998; 82 (Suppl 1): 59-64.

17. Drach GW, Layton TN, Binard WJ. Male peak urinary flow rate: relationships to volume and age. J Urol 1979; 122 (2): 210-214.

18. Homma Y, Imajo C, Takabashi S, Kawabe K, Aso Y. Urinary symptoms and urodynamics in a normal elderly population. Scand J Urol Nephrol Suppl 1994; 157: 27-30.

19. Englesbe MJ, Terjimanian MN, Lee JS et al. Morphometric age and surgical risk. J Am Coll Surg 2013; 2016 (5): 976-985.

20. Thuret R, Hurel S, Kleinclauss F, Timsit MO. Lower urinary tract symptoms and pelvic floor dysfunction in renal transplant candidates and recipients. Prog Urol 2016; 26 (15): 1114-1121.

21. Lubetzky M, Ajaimy M, Kamal L, deBoccardo G, Akalin E, Kayler L. Kidney transplant complications from undiagnosed benign prostatic hyperplasia. Clin Transplant 2015; 29 (6): 539-542.

22. Hurst FP, Neff RR, Falta EM et al. Incidence, predictors, and associated outcomes of prostatism after kidney transplantation. Clin J Am Soc Nephrol 2009; 4 (2): 329-336.

23. Fockens MM, Alberts VP, Bemelman FJ, Laguna Pes MP, Idu MM. Internal or External Stenting of the Ureterovesical Anastomosis in Renal Transplantation. Urol Int 2016; 96 (2): 152-156.

24. Wu X, Dong Y, Liu Y et al. The prevalence and predictive factors of urinary tract infection in patients undergoing renal transplantation: A metaanalysis. Am J Infect Control 2016; 44 (11): 1261-1268.

Received August 20, 2020. Accepted November 18, 2020. 